\title{
Familial and primary (AL) cardiac amyloidosis: echocardiographically similar diseases with distinctly different clinical outcomes
}

\author{
Simon W Dubrey, Karen Cha, Martha Skinner, Michael LaValley, Rodney H Falk
}

\begin{abstract}
Objective-To determine whether patients with myocardial amyloidosis due either to AL (primary) amyloid or familial amyloid have distinguishing echocardiographic or electrocardiographic features; and to compare the prevalence of heart failure and survival in the two types of amyloidosis in relation to echocardiographic findings.
\end{abstract}

Design-Blinded group comparison of randomly selected cases of cardiac amyloidosis.

Setting-International referral centre for amyloid research and treatment.

Patients-36 patients with cardiac amyloid heart disease, of whom 12 had familial and 24 had primary AL amyloidosis.

Results-Familial and AL echocardiograms were morphologically indistinguishable, with similar left ventricular wall thickness, mean (SD) $15.4(2.3)$ v 15.8 $(2.5) \mathrm{mm}$, respectively; right ventricular wall thickness was also similar between amyloid types: $9.6(2.8)$ v $9.7(6.5) \mathrm{mm}$, respectively. Doppler indices of left and right ventricular function, left ventricular volume, and ejection fraction were also similar. Low voltage electrocardiograms (< $0.5 \mathrm{mV}$ ) were more common in the $A L$ $(16 / 24,67 \%)$ than in the familial group $(4 / 12,25 \%), P<0.05$. The one year survival for familial and $A L$ forms was $92 \%$ (11/12) $v$ $38 \%(6 / 24)$, respectively, with virtually all deaths due to cardiac causes.

Conclusions-Although cardiac involvement is echocardiographically indistinguishable, cardiac mortality is very different between the two forms of amyloidosis. Preservation of electrocardiographic voltage in familial amyloidosis suggests that the particular biochemical characteristics of distinct types of amyloid fibril have different pathological effects on the myocardium. This distinction becomes critical in the evaluation, treatment, and management of patients who have a diagnosis within the spectrum of the protein deposition diseases.

(Heart 1997;78:74-82)

Keywords: amyloidosis; echocardiography; familial amyloidosis

Familial amyloid polyneuropathy (FAP), identified just over 40 years ago, ${ }^{1}$ is the rarest type of systemic amyloidosis. ${ }^{2}$ The exact prevalence of
FAP is unknown but, based on referral patterns to major centres specialising in amyloidosis, it probably represents $10 \%$ of patients with the disease. It is estimated that there are between 1000 and 2225 new cases of AL (primary) amyloidosis in the United States each year, ${ }^{34}$ and therefore one might predict between 100 to 200 newly diagnosed cases of familial amyloidosis per year. A transthyretin mutant protein produced by the liver is responsible for most cases of familial amyloidosis, and the resultant protein deposition may cause dysfunction of various organ systems including the peripheral and autonomic nerves, gastrointestinal tract, and heart. ${ }^{2}$ Disorders of conduction necessitating permanent pacing are the predominant manifestation of cardiovascular disease, ${ }^{5}$ but myocardial infiltration with amyloid is also frequently present. ${ }^{6}$

The echocardiographic appearance of myocardial involvement in FAP has been described as similar to that in $\mathrm{AL}$ amyloidosis (light chain amyloidosis, formerly called primary amyloidosis). ${ }^{7}$ Typical abnormalities consist of increased right and left ventricular wall thickness with normal cavity size, increased myocardial echogenicity, and valve thickening. ${ }^{8}$ Data from patients with $\mathrm{AL}$ amyloid indicate that myocardial involvement documented by echocardiography is often accompanied by congestive heart failure and that its presence is associated with a median survival less than six months. ${ }^{4}$ Survival in familial amyloidosis with cardiac involvement appears to be longer than in AL amyloidosis, ${ }^{9}$ although no direct comparison has been made and no attempt has been made to compare groups of patients with similar echocardiographic appearances. Recently liver transplantation has been shown to be an effective treatment for patients with FAP. ${ }^{10}$ By removing the source of the mutant protein the disease is arrested and may even regress. ${ }^{11}$ The significance of cardiac amyloid infiltration in patients being evaluated for liver transplantation is not known. However, since its presence in AL amyloidosis augurs an ominous prognosis, it might be expected to carry a similar prognosis in the familial form, thus rendering the risks of major surgery prohibitive. We therefore undertook this study to determine whether patients with FAP and definite cardiac involvement had echocardiographic features which distinguished them from patients with AL cardiac amyloidosis, and whether the clinical and prognostic significance of such cardiac involvement differed from a cohort of patients with $\mathrm{AL}$ amyloidosis. 


\begin{abstract}
Methods
Over a period of eight years (1986-94), 40 patients with familial amyloidosis and 232 with AL amyloidosis were seen at the clinical research centre of Boston University Medical Center. All patients referred with the diagnosis of amyloidosis undergo an extensive clinical evaluation including a full non-invasive cardiac examination. This includes a 12 lead electrocardiogram, 24 hour Holter recording, and Doppler and cross sectional echocardiography. Following initial evaluation patients are prospectively followed by written contact and annual visits.
\end{abstract}

\section{ECHOCARDIOGRAPHY}

Echocardiograms were performed using a Hewlett Packard phased array system. M mode recordings were made at $50 \mathrm{~mm} / \mathrm{s}$ with simultaneous recording of the electrocardiogram. Heart failure was considered present on physical examination in patients with raised jugular venous pressure or evidence of pulmonary venous congestion or both. Severity of heart failure was classified using the New York Heart Association criteria. ${ }^{12}$ Of these 40 patients with FAP, 17 had no clinical or echocardiographic evidence of cardiac involvement and two had conduction system disease but normal echocardiograms. An additional nine patients had mild left ventricular wall thickening on echo $(\leqslant 1.3 \mathrm{~cm})$ without clinical symptoms of cardiac disease. The remaining 12 patients from the familial group had ventricular wall thickening greater than $1.3 \mathrm{~cm}$ unexplained by hypertension or valve disease, and formed the index group for this study. In order to evaluate similarities and differences between cardiac involvement in patients with FAP and AL amyloidosis we randomly chose 24 patients with $\mathrm{AL}$ amyloidosis from among 133 patients whose echocardiograms showed a wall thickness $>1.3 \mathrm{~cm}$, in the absence of hypertension or significant valve disease. Cardiac involvement by echocardiography was the single criterion for patient recruitment of both groups and selection was blind to any additional clinical features or outcome aside from amyloid type.

DIAGNOSIS OF AMYLOID TYPE

All 36 patients studied had biopsy proven systemic amyloidosis. The diagnosis of AL amyloidosis was made by histological evidence of systemic amyloid in association with evidence of a plasma cell dyscrasia and/or by identification of an immunoglobulin light chain in their serum, urine, or amyloid deposit. None of these 24 patients had evidence of multiple myeloma. The diagnosis of FAP was confirmed by isoelectric focusing of the serum to identify variant transthyretin, which was followed by detection of a mutant transthyretin gene known to be associated with familial amyloidosis identified in their DNA. In all 12, a family history of amyloidosis was also present. The type of transthyretin mutation responsible for amyloid production was available in 37 of the 40 FAP patients $(92.5 \%)$ from whom the study group was derived. The commonest mutation, occurring in 10 of the 40 patients $(25 \%)$, was valine-30-methionine. This mutation was only present in two of the 12 FAP patients $(16.7 \%)$ with significant echocardiographic abnormalities, reflecting the relative infrequency of myocardial infiltration in this genetic form compared with other mutations. The remaining 10 FAP index group patients had the following amino acid substitutions: threonine-60-alanine in two, valine-30-alanine in one, and glutamate-89glutamine in one. Six further patients were identified as having two mutations each, respectively glutamate-42-glycine and histidine-90-asparagine.

SURVIVAL ANALYSIS

Survival was measured from the time of the echocardiogram until death except in four $\mathrm{AL}$ patients who underwent cardiac transplantation. These patients were considered to have fatal organ failure (a death equivalent) from the date of this procedure. Liver transplantation was not considered an end point in patients with FAP, since the liver function in these patients is normal and the purpose of transplantation is to remove the chronic source of mutant protein synthesis. Within the AL group, 11 patients received melphalan and prednisone, 12 received colchicine alone, and one patient had an extremely rapid demise and died before any treatment could be started.

AL amyloid may seriously affect the kidneys as well as the heart and this combination may have a detrimental effect on survival. We addressed any unintentional bias towards more significant renal disease in the $\mathrm{AL}$ group by reanalysing the $\mathrm{AL}$ patients $(\mathrm{n}=16)$ without such renal disease (serum creatinine $<115 \mu \mathrm{mol} / 1$ and 24 hour urinary protein excretion $<3 \mathrm{~g} / 24$ hours on any occasion) and comparing the survival of these $16 \mathrm{AL}$ patients with the familial group. We also compared survival in a subgroup of the AL patients with cardiac amyloidosis who were age matched to the familial group.

\section{ASSESSMENT OF ECHOCARDIOGRAMS}

This was performed for all 36 echocardiograms, in a blinded fashion, to determine if there were features that were specific to the individual types of amyloidosis. Two experienced cardiologists examined the echocardiograms; both were unaware of the type of amyloid disease. For all patients a consensus opinion was reached regarding the presence of the classical features originally described in $\mathrm{AL}$ cardiac amyloidosis ${ }^{4}$ : myocardial thickening and increased echogenicity ("granular sparkling"), valve thickening, and interatrial septal thickening. Additional features previously described in amyloid heart disease, including pericardial effusion and atrial dilatation, were also recorded. Left ventricular systolic performance was judged to be normal (ejection fraction $\geqslant 55 \%$ ), mildly impaired (ejection fraction of $40-54 \%$ ), moderately impaired (ejection fraction $30-39 \%$ ), or severely impaired (ejection fraction $<30 \%$ ).

In addition to blinded qualitative assess- 


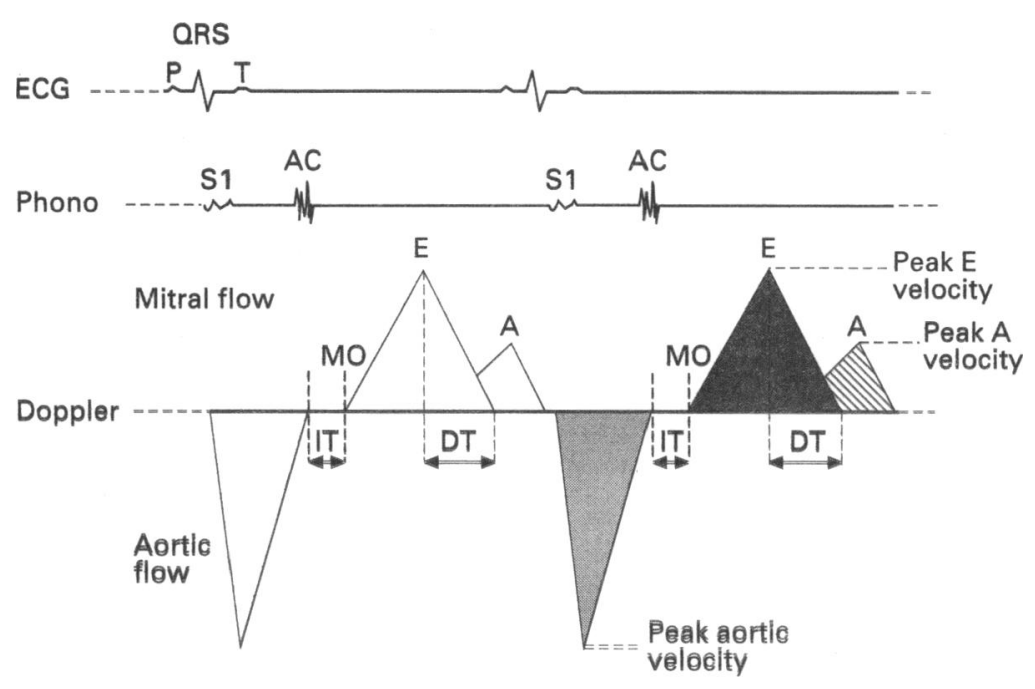

Figure 1 Illustration of Doppler measurements and intervals. Aortic flow is represented as the waveform below the baseline. AC represents aortic valve closure; $D T$, the deceleration time of the early filling phase; S1, the first heart sound; IT, the left ventricular

isovolumetric relaxation time, measured from the aortic valve closure $(A C)$ to the start of mitral flow (MO). The shaded areas of the mitral waveform indicate the waveform proportions used in the measurement of the $E$ and $A$ wave velocity time integrals.

ment, quantitative measurements of all echocardiograms were made by a single reader. Left ventricular fractional shortening was calculated as diastolic minus systolic left ventricular internal dimension divided by diastolic internal dimension. Right ventricular wall thickness was measured from $M$ mode recordings obtained from a subcostal four chamber view. Cardiac output was calculated from the product of the left ventricular outflow tract area, the area of the Doppler systolic aortic velocity time integral, and the heart rate-the latter derived from the immediate preceding $\mathbf{R}-\mathbf{R}$ interval.

Pulsed Doppler left ventricular outflow was obtained in the apical five chamber view, with the sample volume placed approximately $5 \mathrm{~mm}$ proximal to the aortic valve leaflets. Mitral and tricuspid Doppler studies were performed at the level of the leaflet tips, and flow velocity waveforms were analysed to determine if restrictive ventricular filling patterns were present. ${ }^{13} \mathrm{~A}$ pattern of restrictive haemodynamics to left ventricular inflow was defined as one that satisfied all of the following three criteria: an $E$ wave deceleration time of $<150 \mathrm{~ms}^{14}$ an $\mathrm{E}$ to $\mathrm{A}$ wave velocity ratio of greater than $2 \cdot 5$, and an $\mathrm{E}$ to $\mathrm{A}$ wave velocity time integral ratio of greater than $4 \cdot 7$. Both ratios are greater than $3 \mathrm{SD}$ from the mean values for a normal population of this age group,,$^{15}$ and fall well within the range of values reported for restrictive cardiomyopathy. ${ }^{13}$ All Doppler spectral velocity tracings were digitised on screen, where the contour of the Doppler spectral display was traced for six individual consecutive beats for the aortic, mitral, and tricuspid valves. Both two dimensional echocardiographic and Doppler measurements were performed off line using a computerised digital analysis system (Cine view plus; Freeland Systems, Broomfield, Colorado, USA). Left ventricular isovolumetric relaxation time was obtained from pulsed wave Doppler interrogation of the left ventric- ular inflow tract velocities and simultaneous phonocardiography to identify the timing of aortic valve closure (fig 1 ). In the absence of a phonocardiogram, continuous wave Doppler was used to measure the left ventricular isovolumetric relaxation time by directing the cursor across the left ventricular outflow and inflow tracts, thus recording the termination of aortic flow and the onset of mitral valve flow. Left ventricular ejection fraction was calculated from tracings of the endocardial border in diastole and systole; volumes were calculated and ejection fractions determined using the computerised digital analysis system previously described.

\section{BLBGTROGARDIOGRAPHY}

Twelve lead electrocardlogram were per= formed on all patients and callbrated to a $10 \mathrm{~mm}$ deflection equivalence to $1 \mathrm{mV}$. A low voltage electrocardiogram was defined as a mean QRS voltage amplitude in the standard and unipolar leads (I, II, III, aVL, and aVF) of $\leqslant 0.5 \mathrm{mV}$. A pseudoinfarction pattern was defined by QS waves in the anteroseptal (V1V3) and/or the inferior leads (II, III, and aVF), in the absence of previous myocardial infarction. Augmented left ventricular voltages were said to be present if the $S$ wave in V1 plus the largest $R$ wave in V5 or V6 exceeded $3.5 \mathrm{mV}$. The duration of the QT interval was corrected for heart rate to produce the Qtc interval.17 Prolongation of the Qte interval was taken as a duration of more than 0.45 seconds for men and 0.47 seconds for women. ${ }^{18}$ Twenty four hour Holter recordings were analysed for the presence of rhythm disturbances and conduction disorders.

Electrocardiographic voltage to left ventricular mass ratio

The voltage to mass ratio was derived from the electrocardiogram voltage both for the precordial leads ( $\mathrm{S}$ wave voltage in V1 plus the mean of the $R$ wave voltage in V5 and V6), ${ }^{19}$ and the limb leads. This was divided by the cross sectional area of the left ventricular wall (CSA) imaged in a transverse plane. ${ }^{20} \mathrm{CSA}$ was calculated from the left ventricular end diastolic dimension (LVEDD), the mean left ventricular wall thickness (Th) at end diastole, and the body surface area (BSA), using the geometric formula:

$$
\begin{gathered}
\mathrm{CSA}=\left[\{(\mathrm{LVEDD} / 2)+\mathrm{Th}\}^{2}-\right. \\
\left.(\mathrm{LVEDD} / 2)^{2}\right] / \mathrm{BSA}
\end{gathered}
$$

In healthy subjects without cardiac amyloidosis normal values for this left ventricular mass equivalent corrected for body surface area are in the range of $6-10 \mathrm{~cm}^{2} / \mathrm{m}^{2}{ }^{20}$

\section{STATISTICAL ANALYSIS}

Values are given as mean (SD). Statistical analyses were carried out using the SAS package (SAS Institute, Cary, North Carolina, USA). Continuous data for Doppler, echocardiographic, and electrocardiographic measurements were tested using an unpaired nonparametric Mann Whitney U test. Fisher's exact test was used for the analysis of discrete 
Table 1 Clinical and laboratory features of the two patient groups with cardiac amyloidosis

\begin{tabular}{|c|c|c|c|}
\hline Features & Familial $(F A P)$ amyloidosis $(n=12)$ & Primary $(A L)$ amyloidosis $(n=24)$ & Pvalue \\
\hline Age (years) & $47(11)$ & $58(11)$ & $<0.01$ \\
\hline & $8 \mathrm{M} / 4 \mathrm{~F}$ & $15 \mathrm{M} / 9 \mathrm{~F}$ & NS \\
\hline Systolic BP (mm Hg) & $114(11)$ & $113(20)$ & NS \\
\hline Diastolic BP (mm Hg) & $72(8)$ & $68(11)$ & NS \\
\hline Heart rate (beats/min) & $83(15)$ & $75(6)$ & NS \\
\hline Total serum protein $(\mathrm{g} / \mathrm{dl})$ & $6 \cdot 1(0 \cdot 7)$ & $5.8(0.8)$ & NS \\
\hline Serum albumin $(\mathrm{g} / \mathrm{dl})$ & $3.7(0.5)$ & $3.5(0.6)$ & NS \\
\hline \multicolumn{4}{|l|}{ CHF-NYHA class ${ }^{\star}$} \\
\hline II & $10(83 \%)$ & $2(8 \%)$ & $<0.0001$ \\
\hline & $2(17 \%)$ & $7(29 \%)$ & NS \\
\hline IV & $\begin{array}{l}0 \\
0\end{array}$ & $11(46 \%)$ & $\begin{array}{l}<0.01 \\
\text { NS }\end{array}$ \\
\hline One year survival & $11(92 \%)$ & $6(25 \%)$ & 0.0002 \\
\hline Median survival (months) & $25 \cdot 0$ & 5.5 & $<0.005$ \\
\hline Sudden death & $2(17 \%)$ & $6(25 \%)$ & \\
\hline Death due to CHF & $0(0 \%)$ & $16(67 \%)$ & $<0.0005$ \\
\hline
\end{tabular}

Data are reported as mean (SD) or No (\%). Median survival is measured from date of the echocardiogram.

BP, blood pressure; CHF, congestive heart failure; NYHA, New York Heart Association classification of heart failure.

$\star_{n}=23$ for primary group as one patient in this group had intermittent symptoms of CHF and angina that was also influenced by concurrent renal dialysis, thus making the classification of NYHA heart failure unreliable in this patient.
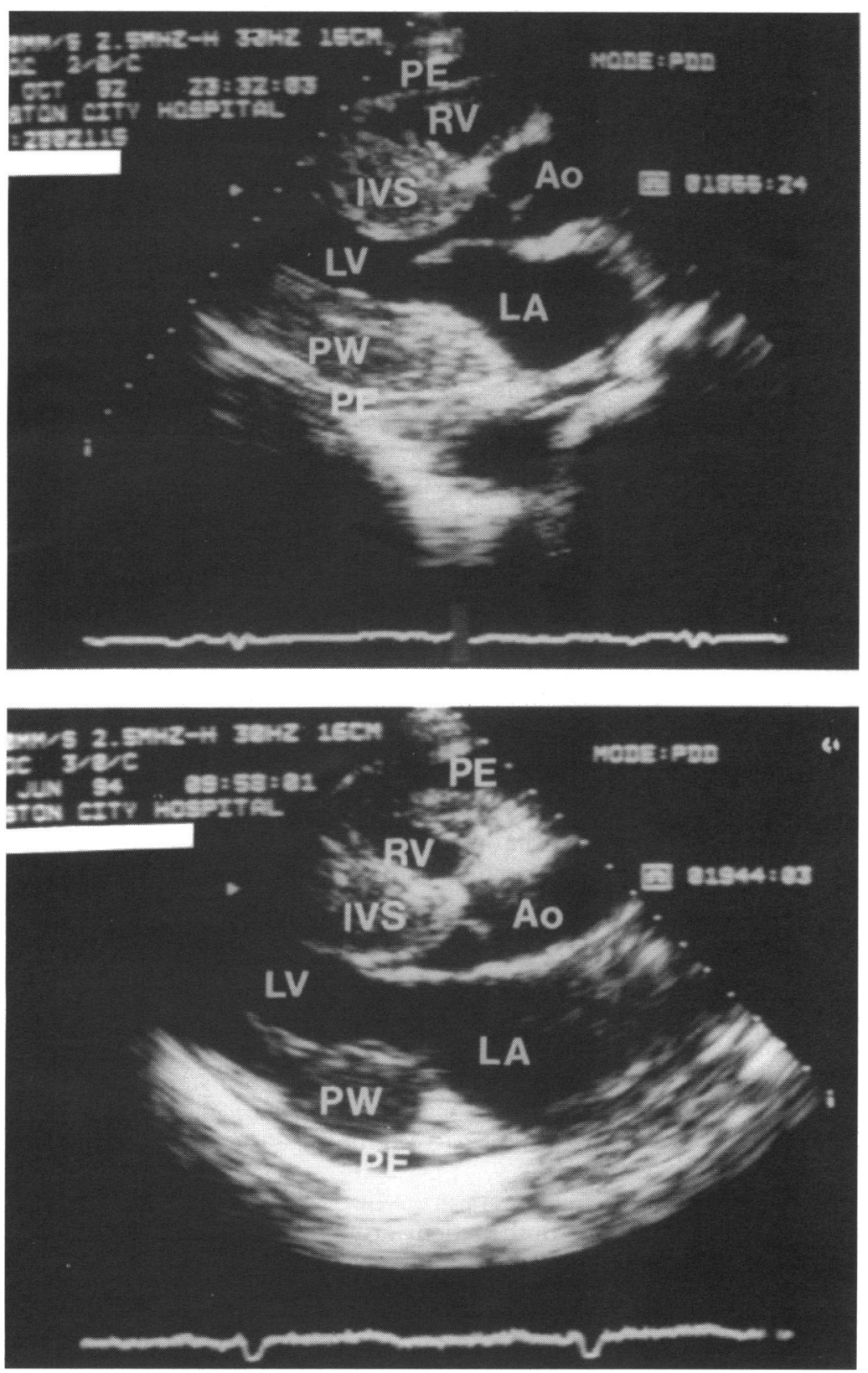

Figure 2 (Top) Parasternal long axis view from a patient with $A L$ cardiac amyloidosis showing left ventricular wall thickening. The left atrium is dilated with thickened mitral valve leaflets and there is a small circumferential pericardial effusion. (Bottom) Parasternal long axis view from a patient with familial amyloidosis showing very similar features of cardiac amyloidosis to those seen in the top panel. Ao, aorta; IVS, interventricular septum; $L A$, left atrium; $L V$, left ventricle; $P E$, pericardial effusion; $P W$, posterior wall; $R V$, right ventricle. data. A univariate survival analysis was performed using the logrank test and data displayed as a Kaplan-Meier plot. No adjustment for multiple comparisons was made; $P<0.05$ was considered to represent significance.

\section{Results}

Twelve patients (eight men, four women) formed the familial group, with a mean age of 47 (11) years. The AL amyloid group consisted of 24 patients (15 men, nine women) with a mean age of 58 (11) years; significantly greater than that of the familial group $(P<$ $0.01)$. All patients were in sinus rhythm. The heart rate and both systolic and diastolic blood pressures were similar for patients with the two types of amyloidosis (table 1). Significant multiorgan involvement was present in eight of 12 FAP patients; six had gastrointestinal and neurological symptoms, while two had gastrointestinal and four had neurological symptoms in addition to their cardiac disease. Renal involvement, with a serum creatinine $>176 \mu \mathrm{mol} / 1$ and/or a 24 hour urine protein $>3 \mathrm{~g}$, was present in eight of the 24 patients with $\mathrm{AL}$ amyloidosis. However serum albumin was not below $2 \mathrm{~g} / \mathrm{dl}$ in any patient.

\section{ECHOCARDIOGRAPHIC DIFFERENCES BETWEEN}

FAP AND AL AMYLOID GROUPS

On blinded review of the echocardiograms, the readers were unable to distinguish $\mathrm{AL}$ amyloidosis from FAP (fig 2) and no differences in echocardiographic features were noted between groups (table 2). In addition to the well described features of cardiac amyloidosis, that is, increased myocardial echogenicity, and thickening of walls, valves, and the atrial septum, ${ }^{719}$ we found that pericardial effusion and atrioventricular valve incompetence was common. Atrioventricular valve incompetence occurred in $92 \%$ (11 of 12) of the familial and $88 \%$ (21 of 24 ) of the AL amyloid groups, with a trend for more of the AL amyloid patient group to have higher grades of tricuspid incompetence (table 2).

Quantitative echocardiographic measurements Mean values for left atrial diameter and interventricular septal, left ventricular posterior 
Table 2 Qualitative echocardiographic features in the two patient groups with cardiac amyloidosis

\begin{tabular}{lccl}
\hline Echocardiographic feature & $\begin{array}{l}\text { Familial (FAP) } \\
\text { amyloidosis } \\
(n=12)\end{array}$ & $\begin{array}{l}\text { Primary (AL) } \\
\text { amyloidosis } \\
(n=24)\end{array}$ & P value \\
\hline Pericardial effusion & $2(17)$ & $8(33)$ & NS \\
Myocardial granular sparkling & $10(83)$ & $21(88)$ & NS \\
Thickened heart valves & $8(67)$ & $16(67)$ & NS \\
Thickened eustachian valve & $5(42)$ & $10(42)$ & NS \\
Thickened interatrial septum & $10(83)$ & $15(63)$ & NS \\
Left atrial dilatation & $8(67)$ & $17(71)$ & NS \\
Bi-atrial dilatation & $5(42)$ & $15(63)$ & NS \\
Valve dysfunction & $3(25)$ & $9(38)$ & NS \\
Mitral regurgitation & $2(17)$ & $10(42)$ & NS \\
Tricuspid regurgitation & $1(8)$ & $0(0)$ & \\
Aortic regurgitation & &
\end{tabular}

Values are No (\%). Valve dysfunction is defined as colour Doppler echocardiographic appearances of regurgitation of $\geqslant$ grade $2 / 4$. Mitral regurgitation of $\geqslant$ grade $3 / 4$ was seen in one familial and one AL patient, tricuspid regurgitation of $\geqslant$ grade $3 / 4$ was not seen in the familial group but was present in five of the AL patients $(P=N S)$.

Table 3 Quantitative echocardiographic measurements in the two patient groups with cardiac amyloidosis

\begin{tabular}{lccc}
\hline Feature & $\begin{array}{l}\text { Familial (FAP) } \\
\text { amyloidosis } \\
(n=12)\end{array}$ & $\begin{array}{l}\text { Primary (AL) } \\
\text { amyloidosis } \\
(n=24)\end{array}$ & P value \\
\hline LA (mm) & $45 \cdot 8(4 \cdot 2)$ & $43 \cdot 7(4 \cdot 7)$ & NS \\
IVS (mm) & $17 \cdot 2(3 \cdot 1)$ & $16 \cdot 5(2 \cdot 6)$ & NS \\
LVPW (mm) & $13 \cdot 7(2 \cdot 3)$ & $15 \cdot 1(2 \cdot 6)$ & NS \\
No of ASH (IVS:LVPW ratio > 1.3) & 1 & 1 & NS \\
No of ASH (IVS: LVPW ratio > 1.5) & 2 & 0 & NS \\
RVWT (mm) & $9 \cdot 6(2 \cdot 8)$ & $9 \cdot 7(6 \cdot 5)$ & NS \\
LVEDD (mm) & $48 \cdot 1(5 \cdot 1)$ & $42 \cdot 5(6 \cdot 4)$ & $<0 \cdot 05$ \\
LVESD (mm) & $32 \cdot 9(6 \cdot 1)$ & $29 \cdot 5(6 \cdot 4)$ & NS \\
FS (\%) & $36 \cdot 3(10 \cdot 7)$ & $29 \cdot 0(9 \cdot 0)$ & $0 \cdot 09$ \\
Ejection fraction data & $58(12)$ & $52(12)$ & NS \\
Mean EF (\%) & $7(58 \%)$ & $8(38 \%)^{\star}$ & NS \\
Normal (EF of > 55\%) & $4(33 \%)$ & $11(52 \%)^{\star}$ & NS \\
Mild-moderate reduction (EF of 40-55\%) & $1(8 \%)$ & $2(10 \%)^{\star}$ & NS \\
Moderate-severe reduction (EF of < 40\%) & $1(8)$ & \\
\hline
\end{tabular}

Data are presented as mean (SD) or No (\%).

ASH, asymmetric septal hypertrophy, quoted for two cut off ratios; EF, ejection fraction; FS, fractional shortening; IVS, interventricular septum; LA, left atrium; LVEDD, left ventricular end diastolic diameter; LVESD, left ventricular end systolic diameter; LVPW, left ventricular posterior wall; RVWT, right ventricular wall thickness.

Numbers and percentages are for a total of $21 \mathrm{AL}$ amyloid patients who had satisfactory echocardiograms for digitised calculation of the ejection fraction to be performed.

Table 4 Doppler measurements in the two patient groups with cardiac amyloidosis

\begin{tabular}{|c|c|c|c|}
\hline Feature & $\begin{array}{l}\text { Familial (FAP) } \\
\text { amyloidois } \\
(n=12)\end{array}$ & $\begin{array}{l}\text { Primary }(A L) \\
\text { amyloidosis } \\
(n=24)\end{array}$ & $P$ value \\
\hline \multicolumn{4}{|l|}{ Mitral valve } \\
\hline E:A wave velocity ratio & $4.41(2 \cdot 97)$ & $4 \cdot 69(4 \cdot 19)$ & NS \\
\hline E:A wave VTI ratio & $4.41(2 \cdot 97)$ & $5 \cdot 11(4 \cdot 11)$ & NS \\
\hline DT (ms) & $157(28)$ & $151(28)$ & NS \\
\hline IVRT (ms) & $89(18)$ & $92(25)$ & NS \\
\hline No (\%) with restrictive LV filling & $3(25 \%)$ & $12(50 \%)$ & NS \\
\hline \multicolumn{4}{|l|}{ Tricuspid valve } \\
\hline $\begin{array}{l}\text { E:A wave velocity ratio } \\
\text { Aortic valye }\end{array}$ & $1 \cdot 26(0 \cdot 46)$ & $1.98(1.34)$ & NS \\
\hline \multicolumn{4}{|l|}{ Aortic valve } \\
\hline VTI $(\mathrm{cm})$ & $17 \cdot 78(5 \cdot 34)$ & $14 \cdot 28(4 \cdot 87)$ & $<0.05$ \\
\hline Heart rate (beats/min) & $76(5)$ & $83(14)$ & NS \\
\hline $\begin{array}{l}\text { Stroke volume (ml) } \\
\text { Cardiac output (1/min) }\end{array}$ & $\begin{array}{c}52 \cdot 9(15 \cdot 4) \\
4 \cdot 01(1 \cdot 21)\end{array}$ & $\begin{array}{c}46.0(19.8) \\
3.82(1.69)\end{array}$ & $\begin{array}{l}\text { NS } \\
\text { NS }\end{array}$ \\
\hline
\end{tabular}

Data are presented as mean (SD) or No (\%)

E wave, peak flow velocity in early diastole; A wave, peak flow velocity with atrial contribution in E wave, peak flow velocity in early diastole; A wave, peak flow velocity with atrial contribution in
late diastole; DT, deceleration time of the mitral valve E wave; IVRT, isovolumetric relaxation late diastole; DT, deceleration time of the mitral valve E wave; IVRT, isovolumetric relaxation
time; restrictive LV filling, restrictive left ventricular filling pattern; VTI, flow velocity integral.

wall, and right ventricular wall thickness were similar in the FAP and AL patient groups. Asymmetric septal hypertrophy with an interventricular septum to posterior wall ratio of $>1.3$ was seen in one patient of each group; at a higher threshold ratio of $>1 \cdot 5$, two patients were identified who were both in the familial group. Left ventricular end diastolic dimension was greater in the FAP group (48.1 (5.1) $\mathrm{mm})$ than in the AL group $(42 \cdot 5(6 \cdot 4) \mathrm{mm}$, $P<0.05$ ) (table 3 ). Left ventricular cavity size was in the lower end of the normal range for both groups $^{21}$; no differences were found between the two amyloid groups for the derived ejection fraction (table 3 ).

Doppler evaluation of ventricular inflow, mitral $E$ wave deceleration time and left ventricular isovolumetric relaxation time

Doppler evaluation of mitral valve flow failed to show any differences between groups in A wave velocity, $E$ wave velocity, $E$ to $A$ peak velocity ratio, or ratio of $E$ to $A$ velocity time integrals. Although the mean $\mathrm{E}$ wave and $\mathrm{A}$ wave velocities for the two groups each fell within the normal range, ${ }^{15}$ several individuals in each group had very small $A$ waves, and two patients in the $\mathrm{AL}$ amyloid group had absent $\mathrm{A}$ wave flow on Doppler examination. As a result, mean $\mathrm{E}$ to $\mathrm{A}$ ratios for both groups lay well above the normal range (table 4). ${ }^{15}$

Mitral E wave flow deceleration time was similarly reduced in both the familial (157 (28) $\mathrm{ms}$ ) and AL amyloid groups (151 (28) $\mathrm{ms}$ ) when compared to normal reference values. ${ }^{1316}$ The left ventricular isovolumetric relaxation time was similar in patients from the FAP (89 (18) ms) and the AL patient groups (92 (25) ms). Criteria for a restrictive left ventricular filling pattern on Doppler were satisfied by three of the 12 FAP patients $(25 \%)$ and by 12 of the $24 \mathrm{AL}$ patients $(50 \%)$

Peak tricuspid flow velocity and flow velocity integral in early diastole ( $\mathrm{E}$ wave flow) were similar in both the FAP and AL groups. Similarly, the peak tricuspid flow velocity and flow velocity integral with atrial contraction in late diastole (A wave flow) did not differ between the two groups. The resultant $E$ to $A$ flow velocity ratio was similar in both the FAP $(1.26(0.46))$ and AL groups (1.98 (1.34)).

The flow velocity integral of the aortic outflow Doppler was greater in the FAP patients $(17.78(5.34) \mathrm{cm})$ than in the $\mathrm{AL}$ patients $(14.28(4.87) \mathrm{cm}, \mathrm{P}<0.05)$, although calculated cardiac output did not achieve statistical significance (table 4).

\section{ELECTROCARDIOGRAPHIC DATA}

Common abnormal features included pseudoinfarction and abnormal axis deviation (table 5). Of note, no patient in either group had left bundle branch block. Mean limb lead voltage was lower in the AL patients $(0.42(0.20) \mathrm{mV})$ than in the FAP group $(0.68(0.29) \mathrm{mV}, \mathrm{P}=$ 0.01 ) (figs 3 and 4 ). When expressed as a left ventricular voltage (limb leads) to mass equivalent ratio there remained a significant difference between the FAP $(0.21(0 \cdot 1))$ and AL groups $(0.14(0.06), \mathrm{P}<0.05)$.

\section{TWENTY FOUR HOUR HOLTER DATA}

Both patient groups showed similar numbers of atrial and ventricular premature beats, and episodes of ventricular and supraventricular tachycardia, including atrial fibrillation and flutter (table 5). Two patients in the AL group had had permanent pacemaker implantation; of these one was a patient with second degree atrioventricular block and the second was in a patient who had documented electromechanical dissociation and cardiac arrest during the early stages of a modified exercise stress test. 
Table 5 Values for resting 12 lead and 24 hour Holter electrocardiographic recordings in the two patient groups with cardiac amyloidosis

\begin{tabular}{|c|c|c|c|}
\hline Feature & $\begin{array}{l}\text { Familial (FAP) } \\
\text { amyloidosis } \\
(n=12)\end{array}$ & $\begin{array}{l}\text { Primary }(A L) \\
\text { amyloidosis } \\
(n=24)\end{array}$ & $P$ value \\
\hline $\begin{array}{l}\text { Abnormal ECG } \\
\text { Low voltage ECG }\end{array}$ & $\begin{array}{r}11(92 \%) \\
4(33 \%)\end{array}$ & $\begin{array}{l}22(92 \%) \\
16(67 \%)\end{array}$ & $\begin{array}{l}\text { NS } \\
\text { NS }\end{array}$ \\
\hline $\begin{array}{l}\text { Axis deviation } \\
\text { Pifto }\end{array}$ & & & \\
\hline $\begin{array}{l}\text { Right }\left(+91^{\circ} \text { to }+180^{\circ}\right) \\
\text { Left }\left(-31^{\circ} \text { to }-90^{\circ}\right)\end{array}$ & $\begin{array}{l}4(33 \%) \\
4(33 \%)\end{array}$ & $\begin{array}{l}8(33 \%) \\
8(33 \%)\end{array}$ & $\begin{array}{l}\text { NS } \\
\text { NS }\end{array}$ \\
\hline ECG voltage (limb) (mV) & $0.68(0.29)$ & $0.42(0 \cdot 20)$ & 0.01 \\
\hline LV-CSA $/ B S A\left(\mathrm{~cm}^{2} / \mathrm{m}^{2}\right)$ & $17 \cdot 2(3 \cdot 8)$ & $16.0(2.9)$ & NS \\
\hline ECG voltage (limb): LVMR & $0.21(0.10)$ & $0 \cdot 14(0 \cdot 06)$ & $<0.05$ \\
\hline $\begin{array}{l}\text { Pseudoinfarct pattern - inferior } \\
\text { Pseudoinfarct pattern - anterio }\end{array}$ & $3(25 \%)$ & $6(25 \%)$ & NS \\
\hline $\begin{array}{l}\text { Pseudoinfarct pattern - anterior } \\
\text { Conduction disturbances }\end{array}$ & $6(50 \%)$ & $9(38 \%)$ & \\
\hline $\begin{array}{l}\text { 1st degree AV block } \\
\text { 2nd degree AV block }\end{array}$ & $\begin{array}{l}3(25 \%) \\
0\end{array}$ & $\begin{array}{l}4(17 \%) \\
1(4)\end{array}$ & $\begin{array}{l}\text { NS } \\
\text { NS }\end{array}$ \\
\hline Bifascicular block & $3(25 \%)$ & $2(8 \%)$ & NS \\
\hline 24 Hour Holter data & & & \\
\hline $\begin{array}{l}\text { VVT } \\
\text { SVT }\end{array}$ & $\begin{array}{l}1(8 \%) \\
1(8 \%)\end{array}$ & $\begin{array}{l}1(4 \%) \\
3(13 \%)\end{array}$ & $\begin{array}{l}\text { NS } \\
\text { NS }\end{array}$ \\
\hline Atrial fibrillation or flutter & $\begin{array}{l}1(8 \%) \\
2(17 \%)\end{array}$ & $3(13 \%)$ & NS \\
\hline Atrial norillation or nutter & $2(17 \%)$ & & \\
\hline
\end{tabular}

Data are reported as mean (SD) or No (\%).

ECG, electrocardiogram; ECG voltage (limb), mean limb lead voltage amplitude; LVCSA/BSA, left ventricular cross sectional area corrected for body surface area; LVMR, left ventricular mass ratio; SVT, supraventricular tachycardia; VT, ventricular tachycardia ( $>5$ beats).

In the FAP group two patients also required permanent pacemakers, the indications in both cases being symptomatic bradycardia with syncope.

\section{SURVIVAL CHARACTERISTICS}

Patients in the FAP group had a 92\% (11 of 12) one year survival from the date of their echocardiogram, compared to $25 \%$ (six of 24) for those patients in the AL group $(P=$ 0.0002 ) (table 1). Reference to the KaplanMeier survival analysis (fig 5) shows that prolonged survival was frequent in the FAP
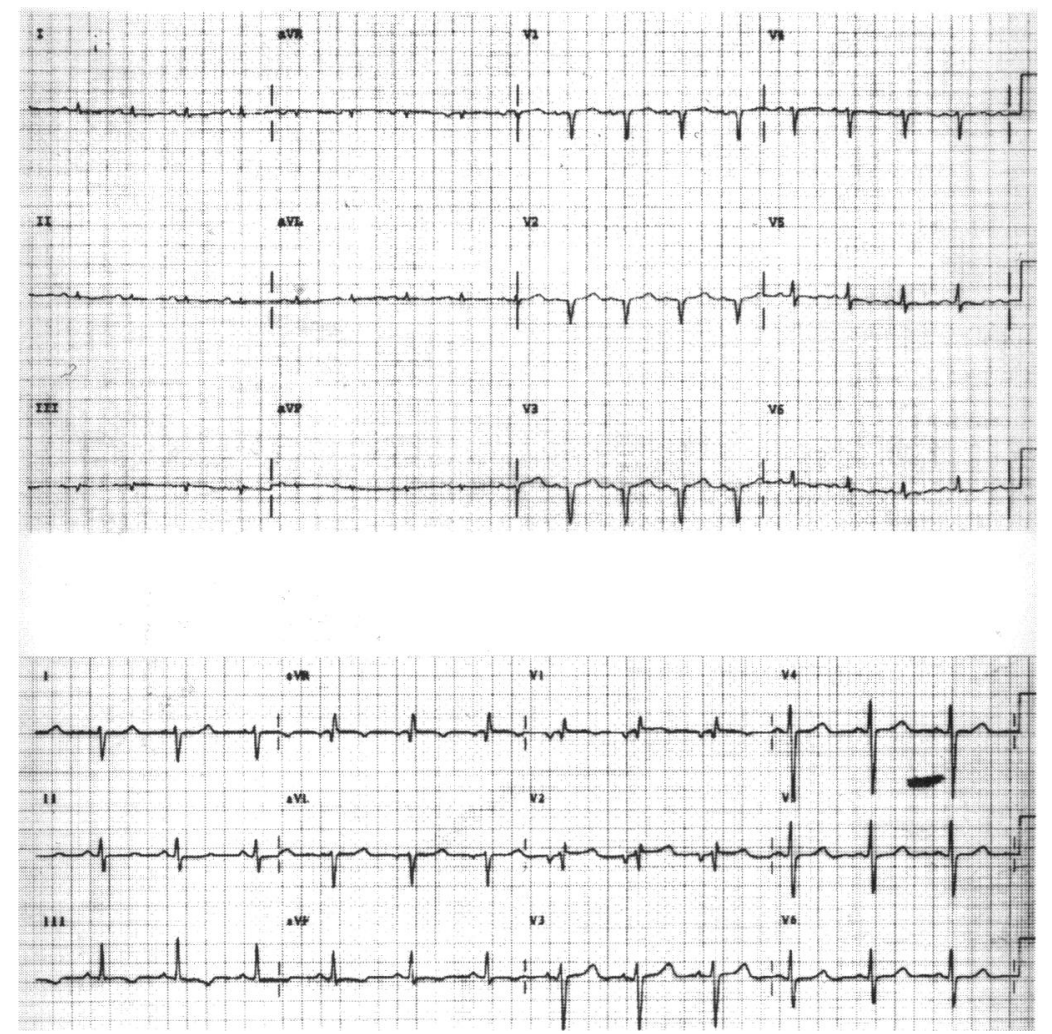

Figure 3 (Top) Resting 12 lead electrocardiogram from the same patient as in fig 2 (top). Note the low voltage complexes, particularly in the limb leads, and a pseudoinfarction pattern of $Q S$ waves in leads VI to V3. (Bottom) Resting 12 lead electrocardiogram from the same patient as in fig 2 (bottom). Note that in spite of similar echocardiographic appearances between the patients shown in fig 2 , the electrocardiographic voltage in the familial patient is greater.

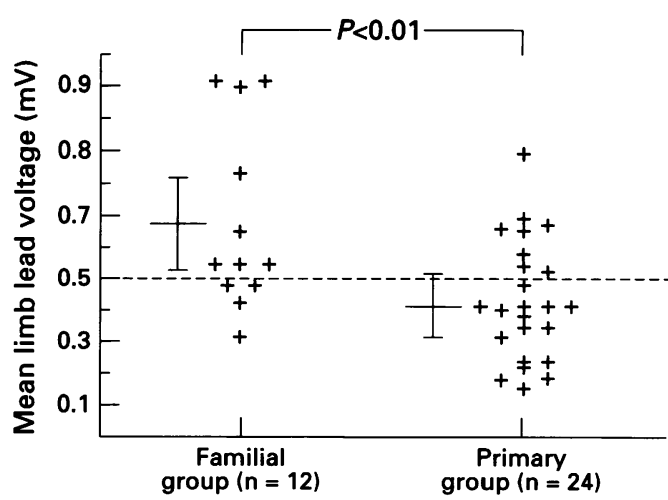

Figure 4 Limb lead voltages for individual patients with familial and AL amyloidosis. The horizontal broken line indicates the defined threshold for low voltage $(<0.5 \mathrm{mV})$. Although there is a considerable overlap of data points, the mean difference in voltage is highly significant $(P=0.01)$.

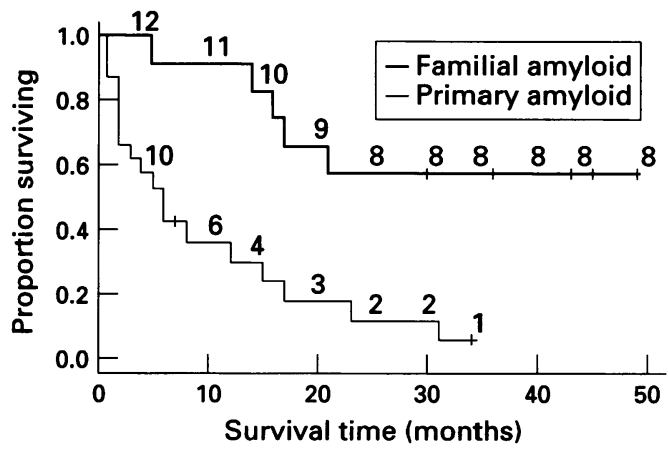

Figure 5 Kaplan-Meier plot showing survival of the familial and AL amyloid patient groups. Death and heart transplantation are treated as equivalent outcomes. The absolute numbers of patients remaining alive at each five month interval are shown on both survival curves. The difference in survival between these two patient groups was highly significant $(P<0.0002)$.

patients and not in the $A L$ patients $(P<$ 0.0002 ). One AL patient died of hepatic failure and the remaining 22 deaths in this group were cardiac: sudden in six and from congestive heart failure in 16. Of the five deaths in the FAP patients, one was caused by sepsis, one was due to inanition, and three were possibly cardiac - two being sudden and one followed several hours of breathlessness of unknown aetiology (table 1 ). The seven surviving FAP patients were followed for a mean of 36 months (range 23 to 50 months) after the qualifying echocardiogram. None of these patients developed class III or class IV heart failure.

Despite the finding of identical echocardiographic appearances, a larger proportion of patients in the AL group (91\%; 21 of 23 ) had congestive heart failure symptoms (New York Heart Association class II to IV) than in the familial group (17\%; two of 12 ), $P<0.0001$. Of these, the two FAP patients were confined to class II symptoms, whereas 14 patients in the AL group had class III-IV heart failure (table 1).

In order to investigate an inadvertent selection bias towards older or sicker $\mathrm{AL}$ patients we analysed survival among an additional group of AL patients who were age matched to 
the FAP group as well as to a subgroup of 16 patients without renal disease from our original study group of $24 \mathrm{AL}$ patients. Twelve AL patients of mean age 47 (12) years and with a wall thickness of $>1.3 \mathrm{~cm}$ (mean $1.61(0.36)$ $\mathrm{cm}$ ) had a median survival of 5.5 months, and in addition only $33 \%$ of these patients survived more than one year from the date of their echocardiogram. Compared to the FAP group who had a one year survival of $92 \%$, survival was significantly shorter in the age matched AL patients $(P=0.009)$. When the one year survival of the FAP group (92\%) is compared to that of the $16 \mathrm{AL}$ patients without renal involvement, of whom four survived one year (25\%), there was a significantly worse survival in the AL patients $(P=0.0006)$.

\section{LIVER TRANSPLANTATION}

Liver transplants were successfully performed in six of the 12 patients with FAP. Despite the extensive myocardial infiltration on echocardiography, no patient had complications of perioperative or postoperative congestive heart failure.

\section{Discussion}

QUALITATIVE ECHO DOPPLER OBSERVATIONS

ON FAP AND AL PATIENT GROUPS

This study shows that the echocardiographic features in AL amyloidosis and FAP were present to a very similar degree in both types of amyloidosis, such that the type of amyloid could not be identified from the echocardiogram.

Klein et al have shown that heart failure and survival in AL amyloidosis is correlated with the degree of myocardial thickening seen on echocardiography. ${ }^{14}$ Our data support this finding in patients with $\mathrm{AL}$ amyloidosis, but show that heart failure may be minimal or absent despite extensive wall thickening in patients with the familial disease. No patient with FAP developed severe heart failure (class III or IV) and no familial patient died of chronic heart failure during the follow up period. Thus the prognosis in FAP, unlike that in light chain associated AL amyloid, cannot be predicted from the echocardiogram.

The finding of a "restrictive" Doppler pattern in three of the 12 FAP patients in the absence of heart failure appears, at first glance, difficult to explain. However a major criterion of the restrictive pattern is a small $A$ wave in conjunction with an abbreviated $E$ wave deceleration time. ${ }^{13}$ Patients with FAP may have other reasons for a small A wave-specifically atrial amyloid, resulting in its most extreme form in electromechanical dissociation of the atrium. ${ }^{22}$ It is possible therefore that this Doppler appearance may not reflect increased atrial afterload but rather decreased atrial contractility.

Liver transplantation, by removing the source of the mutant protein, has become an effective treatment for preventing production of familial amyloid protein (transthyretin) and arresting the disease. ${ }^{11}$ Support for the concept that wall thickening in familial amyloid is of little prognostic significance comes from the response of the six patients who underwent liver transplantation without perioperative problems of fluid balance. Our selection criteria for liver transplantation in this condition exclude patients who are seriously incapacitated, for example from neuropathy, inanition, or severe heart failure. It is of note that we did not have to exclude anyone in the latter category, despite the echocardiographic appearances. We do not believe that liver transplantation had any effect in prolonging survival in patients in this study, since follow up was relatively short and all patients accepted for liver transplantation were expected to live at least five years, based on clinical status.

The two groups had significantly different periods of survival. Although it might be argued that this may be related to the differences in the severity of heart failure at the time of the echocardiogram, we believe that the differences in mortality and in heart failure are intimately linked and reflect differing effects of $\mathrm{AL}$ and FAP amyloid infiltration of the myocardium. The onset of heart failure in patients with $\mathrm{AL}$ amyloid is predicted by the severity of myocardial thickening on echocardiography. ${ }^{14}$ If the same were true in FAP one would anticipate a similar prevalence of heart failure given the similar increase in left ventricular mass, yet heart failure was uncommon, never severe, and did not develop de novo during a mean follow up of 36 months in either the liver transplanted or the non-transplanted group. Furthermore, there is no described phase of myocardial infiltration in AL amyloidosis characterised by marked left ventricular thickening without significant heart failure, so it cannot be argued that the $\mathrm{AL}$ patients were at a later, symptomatic, stage of their cardiac disease than the familial group. We thus believe that these differences in symptoms and mortality reflect true differences in the response of the heart to deposition of amyloid fibrils in the two forms of the disease-a theory that is supported by our observations on the electrocardiographic voltage response.

\section{ELECTROCARDIOGRAPHIC VOLTAGE AND}

VOLTAGE MASS

In general increased cardiac mass is associated with an increase in electrocardiographic voltage. In $\mathrm{AL}$ amyloidosis, there is an inverse relation between left ventricular mass and electrocardiographic voltage. ${ }^{23}$ This is due to a combination of replacement of some myocardial cells by electrically inert amyloid and destruction of many remaining myocytes. Our findings of low limb lead voltage electrocardiograms in $33 \%$ of the familial and $67 \%$ of the AL patients are consistent with previous studies and represents amyloid infiltration of the myocardium. ${ }^{24-26}$ However, there were differences between the two groups which suggested that more functional myocardium remained in the FAP patients. The difference in the mean limb lead voltage between the two groups was statistically significant, as was the difference in the voltage to mass ratio. Although a low volt- 
age recording may be associated with pericardial effusions it seemed unlikely that the small effusions observed in our patients would account for the voltage differences. Indeed analysis of the electrocardiograms of those patients with $(n=8)$ and without $(n=16)$ effusions in the $\mathrm{AL}$ amyloid group showed no significant differences in voltages.

Although the precise mechanism for the relatively preserved voltage in FAP is not clear, it may be postulated that it is related to different histological deposition patterns in the two disease types (for example, lesser physical distortion and destruction of the remaining myocytes in FAP), differences in the biological characteristics of the protein, or differences in myocyte response to myocardial infiltration (for example, reactive hypertrophy of the remaining myocardium in FAP). Such differences, if present, may explain the lesser degrees of heart failure and the better survival in the familial group.

\section{LIMITATIONS OF THE STUDY}

The number of patients with FAP and echocardiographic abnormalities studied was small and thus minor differences between groups may not have been detected. However, the very poor survival in the AL patients is consistent with similar patients in our large $\mathrm{AL}$ patient database and with data from other centres. ${ }^{4}$ The strikingly better survival in the FAP group is highly significant and consistent with other reports of a longer duration of survival in this disease compared to $\mathrm{AL}$ amyloidosis. As indicated in the methods section, the small number of FAP patients studied represents the total number of patients with echocardiographic abnormalities out of a series of 40 patients. Given the extreme rarity of FAP, we believe that these numbers represent one of the largest series of patients with this condition seen in the United States.

\section{CLINICAL IMPLICATIONS}

We have shown that despite very similar echocardiographic appearances, cardiac involvement in FAP and AL amyloidosis has a very different outcome. The clinical significance of these findings is important. If a diagnosis of amyloidosis is suspected by echocardiography and confirmed by tissue biopsy, it is critical to determine the type of amyloid, not only because the prognosis of the heart disease differs but also because the treatment of the two forms is completely different.

\section{Determining the type of amyloidosis}

It is rarely necessary to perform endomyocardial biopsy, and histology is usually obtained from an abdominal fat biopsy-the characteristic apple green birefringence seen when the tissue is stained with congo red and viewed under polarised light is diagnostic for systemic amyloidosis of either the AL or FAP type. After a positive tissue biopsy is obtained and if there is no family history of amyloidosis, $\mathrm{AL}$ amyloidosis is considered first as it is the most common type. A search for a clonal plasma cell dyscrasia is the first step. Monoclonal immunoglobulins or light chains are detected in $90 \%$ of $\mathrm{AL}$ patients by means of immunofixation electrophoresis of serum and urine, a more sensitive technique than simple protein electrophoresis. Patients with apparent $\mathrm{AL}$ amyloidosis who do not have monoclonal light chains can pose a diagnostic problem. In most of these patients, a clonal dominance of plasma cells will be identified by examining a bone marrow biopsy with immunohistochemical staining or by cellular studies employing labelled antibodies specific for human light chains. In rare cases gene rearrangement studies may be employed. When there is no evidence of a plasma cell dyscrasia, consideration should be given to another form of amyloidosis. While a family history of amyloidosis or unexplained progressive neuropathy strongly suggests FAP, a variant transthyretin is sought in all patients who do not have a plasma cell dyscrasia. Transthyretin can be identified by isoelectric focusing of the serum, which will separate variant and wild type transthyretin. The finding of a variant transthyretin in serum then prompts specific genetic testing to define the mutation precisely.

\section{Treatment of AL amyloidosis and FAP}

The treatment of $\mathrm{AL}$ amyloidosis usually involves oral chemotherapy with alkylating agents such as melphalan coupled with prednisolone. Most recently high dose intravenous melphalan has been used in an attempt to annihilate the plasma cell clone; this treatment method requires autologous stem cell rescue to repopulate the bone marrow after the chemotherapy has been given. In the case of FAP, where the abnormal protein is transthyretin, no drug treatment has proved effective and the treatment of choice is liver transplantation to remove the source of the mutant protein.

Our data indicate that in FAP there is a disparity between severe myocardial thickening and clinical features of heart failure, and they suggest that, in the absence of severe heart failure, major surgical procedures may be successfully undertaken. Thus once FAP is diagnosed the finding of echocardiographic abnormalities should not be taken as a sign of impending heart failure or as an absolute contraindication to liver transplantation.

An unanswered question is the reason for the clinical differences in AL amyloidosis and FAP with severe echocardiographic abnormalities. While the electrocardiographic data suggest more residual functional myocardium in FAP, the exact reason awaits a careful histological comparison in the two patient groups and an assessment of the effects of the different biological composition of the amyloid fibrils on normal myocytes.

Supported by NIH Grants 40414, 20613, RR 533, the Sue Sellors Finley Memorial Amyloid Research Fund, and the Amyloid Research Fund of Boston University. We thank Ceit $\mathrm{McC}$ aleb for her invaluable secretarial support.

1 Andrade C. A peculiar form of peripheral neuropathy Familial atypical generalised amyloidosis with special involvement of the peripheral nerves. Brain 1952;75 408-27.

2 Benson MD. Amyloidosis. In: Scriver CR, Beaudet AK, 
Sly WS, Valle D, eds. The metabolic and molecular basis of inherited disease. New York: McGraw-Hill, 1995: of inherited

3 Simms RW, Prout MN, Cohen AS. The epidemiology of AL and AA amyloidosis. Balliere's Clin Rheumatol 1994;8: AL and $A A$

4 Kyle RA, Gertz MA. Primary systemic amyloidosis: clinical and laboratory features in 474 cases. Semin Hematol 1995;32:45-59.

5 Olofsson BO, Andersson R, Furberg B. Atrioventricular and intraventricular conduction in familial amyloidosis with polyneuropathy. Acta Med Scand 1980;208:77-80.

6 Eriksson P, Backman C, Bjerle P, Eriksson A, Holm S, Olofsson BO. Non-invasive assessment of the presence and severity of cardiac amyloidosis. A study in familial amyloidosis with polyneuropathy. Br Heart $\mathcal{f} 1984 ; 52$ : 321-6.

7 Cueto-Garcia L, Reeder GS, Kyle RA, Wood DL, Seward JB, Naessens J, et al. Echocardiographic findings in systemic amyloidosis: spectrum of cardiac involvement and temic amyloidosis: spectrum of cardiac involvement

8 Backman C, Olofsson BO. Echocardiographic features in familial amyloidosis with polyneuropathy. Acta Med Scand 1983;214:273-8.

9 Gertz MA, Kyle RA, Thibodeau SN. Familial amyloidosis: a study of 52 North American-born patients examined during a 30 year period. Mayo Clin Proc 1992;67:428-40.
0 Holmgren G, Ericzon BG, Groth CG, Steen L, Suhr O, Holmgren G, Ericzon BG, Groth CG, Steen L, Suhr O,
Andersen O, et al. Clinical improvement and amyloid regression after liver transplantation in hereditary transthyretin amyloidosis. Lancet 1993;341:1113-6. 11 Steen L, Holmgren G, Suhr O, Wikstrom L, Ericzon BG, patients with familial amyloidotic polyneuropathy. Amyloid: Int ₹ Exp Clin Invest 1994;1:138-42.

12 Criteria Committee, New York Heart Association. Diseases of the heart and blood vessels. Nomenclature and criteria for diagnosis, 6th ed. Boston: Little, Brown and Co, 1964: 114.

13 Appleton CP, Hatle LK, Popp RL. Demonstration of restrictive ventricular physiology by Doppler echocardiography. F Am Coll Cardiol 1988;11:757-68.

14 Klein AL, Hatle LK, Burstow DJ, Seward JB, Kyle RA, Bailey KR, et al. Doppler characterization of left ventricu- lar diastolic function in cardiac amyloidosis. $f \mathrm{Am}$ Coll Cardiol 1989:13:1017-26.

15 Benjamin EJ, Levy D, Anderson KM, Wolf PA, Plehn JF, Evans JC, et al. Determinants of Doppler indexes of left ventricular diastolic function in normal subjects (The Framingham Heart Study). Am $f$ Cardiol 1992;70: 508-15.

16 Klein AL, Cohen GI. Doppler echocardiographic assessment of constrictive pericarditis, cardiac amyloidosis, and cardiac tamponade. Cleveland Clin $\mathcal{F}$ Med 1992;59: 278-90

17 Bazett HC. An analysis of the time relations of electrocardiograms. Heart 1920;7:352-70.

18 Moss AJ. Measurement of the QT interval and the risk associated with QTc interval prolongation: a review. $A m \mathcal{f}$ Cardiol 1993;72:23-5B.

19 Falk RH, Plehn JF, Deering T, Schick EC, Boinay P, Rubinow A, et al. Sensitivity and specificity of the echocardiographic features of cardiac amyloidosis. $A m \mathcal{F}$ Cardiol 1987;59:418-22.

20 Simons M, Isner J. Assessment of relative sensitivities of noninvasive tests for cardiac amyloidosis in documented noninvasive tests for cardiac amyloidosis in docum.
cardiac amyloidosis. Am $₹$ Cardiol 1992;69:425-7.

21 Wahr DW, Wang YS, Schiller NB. Left ventricular volumes determined by two-dimensional echocardiography in a normal adult population. $₹ \mathrm{Am}$ Coll Cardiol 1983; in a norme-8.

22 Dubrey S, Pollak A, Skinner M, Falk RH. Atrial thrombi occurring during sinus rhythm in cardiac amyloidosis: evidence for atrial electro-mechanical dissociation. $\mathrm{Br}$ Heart $\mathcal{F}$ 1995; 74:541-4.

23 Carroll JD, Gaasch WH, McAdam KPWJ. Amyloid cardiomyopathy: characterisation by a distinctive voltage mass relation. Am $₹$ Cardiol 1982;49:9-13.

24 Roberts WC, Waller BF. Cardiac amyloidosis causing cardiac dysfunction: analysis of 54 necropsy patients. $A m \mathcal{F}$ Cardiol 1983;52:137-46.

25 De Freitas AF. The heart in Portuguese amyloidosis. Postgraduate Med $\mathcal{\Im}$ 1986;62:601-5.

26 Hamer JPM, Janssen S, Van Rijswijk MH, Lie KI. Amyloid cardiomyopathy in systemic non-hereditary amyloidosis. cardiomyopathy in systemic non-hereditary amyloidosis. findings in 30 patients with $\mathrm{AA}$ and 24 patients with $\mathrm{AL}$ amyloidosis. Eur Heart $f$ 1992;13:623-7. 